\title{
Transcranial Doppler Monitoring During Laparoscopic Anterior Lumbar Interbody Fusion
}

\author{
Maria J. Colomina, MD*, Carmen Godet, MD*, Ferran Pellisé, MDt, Joan Bagó, MDt, and \\ Carlos Villanueva, MD+
}

Departments of *Anesthesiology and +Spine Surgery, Area de Traumatología, Hospital Universitario Vall d'Hebron, Barcelona, Spain

\begin{abstract}
We studied the consequences on cerebral hemodynamics of lengthy laparoscopic procedures requiring pneumoperitoneum and head-down positioning. From October 1995 to April 1999, 17 ASA status I or II patients (16 women and 1 man; mean age, 38 yr) were treated with laparoscopic anterior lumbar fusion. Besides standard perioperative monitoring for laparoscopic surgery, the mean blood-flow velocity of both middle cerebral arteries and the pulsatility index were determined by transcranial Doppler ultrasound. Adequate acoustic windows were encountered in 11 of the 17 patients, and the remaining 6 were excluded from the analysis. $\mathrm{PaCO}_{2}$ and end-tidal $\mathrm{CO}_{2}$ were maintained within normal limits $(<40 \mathrm{~mm} \mathrm{Hg})$; ventilation was optimized in all cases.
\end{abstract}

There was a significant increase $(P<0.05)$ in heart rate and central venous pressure with the change from supine to head-down position in all patients. Transcranial Doppler results for mean middle cerebral artery bloodflow velocity and pulsatility index showed no significant variations at any of the four time points studied during the procedure. There were no technique-related complications, except for moderate postoperative headache in eight patients that resolved with rest and oxygen therapy. We conclude that lengthy laparoscopic procedures in the head-down position performed in otherwise healthy patients do not significantly affect intracranial circulation.

(Anesth Analg 2003;97: aparoscopic surgery requires the establishment of artificial pneumoperitoneum by gas insufflation (usually $\mathrm{CO}_{2}$ ) with controlled intraabdominal pressure (IAP). This implies cardiocirculatory, respiratory, and metabolic changes, which have been extensively studied (1). Nevertheless, the repercussions of the pneumoperitoneum on cerebral homeostasis and intracranial pressure (ICP) have not been thoroughly assessed.

In October 1995, after completing a training period with animals (30- to $50-\mathrm{kg}$ pigs), our group initiated the use of a laparoscopic technique for anterior L5-S1 interbody fusion in the head-down (Trendelenburg) operating position. The mean duration of the procedure was $360 \mathrm{~min}$, with a total head-down time of approximately $240 \mathrm{~min}$. This study determined the consequences of pneumoperitoneum and lengthy head-down positioning on cerebral hemodynamics in

Accepted for publication July 11, 2003.

Address correspondence and reprint requests to Maria J. Colomina, MD, C/ Rosselló, 72, 3ํ-1, 08029 Barcelona, Spain. Address e-mail to26750mjc@comb.es.

DOI: 10.1213/01.ANE.0000087880.88858.72 patients having laparoscopic lumbar fusion, by monitoring middle cerebral artery flow (MCA) velocity with transcranial Doppler ultrasound.

\section{Methods}

From October 1995 to April 1999, 17 patients diagnosed with L5-S1 discopathy were treated with anterior interbody fusion by using a laparoscopic approach and a head-down operating position. The guidelines for new surgical techniques required by Spanish laws and our institution were followed, and an informed consent form was signed by all patients before surgery (2).

The series included 16 women and 1 man, with mean age $38 \mathrm{yr}$ (range, $28-44 \mathrm{yr}$ ), weight $63 \mathrm{~kg}$ (range, $52-72 \mathrm{~kg}$ ), height $164 \mathrm{~cm}$ (range, 152-173 cm), and ASA physical status I or II. None of the patients had a history of cardiovascular, respiratory, or cerebrovascular disease.

All patients received atropine $0.5 \mathrm{mg}$, fentanyl $0.15 \mathrm{mg}$, midazolam $4 \mathrm{mg}$, and ondansetron $4 \mathrm{mg}$ before surgery. Anesthesia was induced with propofol $2.5 \mathrm{mg} / \mathrm{kg}$ and vecuronium $0.1 \mathrm{mg} / \mathrm{kg}$ IV. Maintenance was provided with a $50 \%$ mixture of oxygen/ 
air, desflurane at a minimum alveolar anesthetic concentration of 0.5 , and continuous perfusions of fentanyl and vecuronium. Normocapnia $\left(\mathrm{PaCO}_{2}\right.$ $\leq 40 \mathrm{~mm} \mathrm{Hg}$ and end-tidal (ET) $\mathrm{CO}_{2} \leq 34-38 \mathrm{~mm} \mathrm{Hg}$ ) was maintained throughout the study in all patients. Mechanical ventilation was adjusted with the volumecontrolled mode (AS3; Datex-Engstroem, Helsinki, Finland) by means of $\mathrm{ETCO}_{2}$ monitoring. Temperature was controlled with an esophageal thermometer, and normothermia $\left(36^{\circ} \mathrm{C}-37^{\circ} \mathrm{C}\right)$ was maintained during the entire procedure by heated blankets (Warm Touch $^{\circledR}$; Mallinckrodt Medical, Inc., St. Louis, MO) and temperature-controlled fluids (Hotline ${ }^{\circledR}$; Rockland, Gilbertsville, PA).

The monitors used included the following.

1. Continuous electrocardiogram (leads DII and V5).

2. Invasive arterial pressure (AP) by cannulation of the radial artery.

3. Central venous pressure (CVP) by right atrial catheterization via the right internal jugular vein.

4. Urinary output.

5. Oxygen saturation $\left(\mathrm{SaO}_{2}\right)$ by pulse oximetry.

6. ETCO $\mathrm{EC}_{2}$ by capnography.

7. AP of carbon dioxide $\left(\mathrm{PaCO}_{2}\right)$.

8. Concentration of desflurane.

Monitoring of right and left MCA blood flow was performed by transcranial Doppler with a $2-\mathrm{MHz}$ pulsed-wave probe (TC-2-64; Eden Medizinische Electronik, Überlingen, Germany). After anesthesia was induced and the patient was positioned, the best transtemporal acoustic window (just above the zygomatic arch) was located on both sides. The probe was fixed to the patient's head with an elastic bandage to maintain a constant angle and avoid errors in the measurement and location of the various portions of the vessel. Doppler signals were measured at a depth of 50-55 mm. Blood-flow velocities were expressed in centimeters per second. The pulsatility index (PI) was calculated according to the formula PI $=$ (peak systolic velocity - minimum diastolic velocity)/mean velocity. Measurements were made by the same observer in all cases. Among the 17 patients monitored, adequate acoustic windows were encountered in 11; the remaining 6 were excluded from analysis.

After the induction of anesthesia and with patients in supine position, baseline measurements were taken (Time 0 of the study). Patients were then placed in a $15^{\circ}-20^{\circ}$ head-down position, and $\mathrm{CO}_{2}$ insufflation was started. IAP was maintained at $10-12 \mathrm{~mm} \mathrm{Hg}$ during the entire procedure. Measurements were taken at Hours 1, 2, and 3 after head-down positioning and at the time when the peritoneal cavity was deflated and patients were returned to the supine position (end of surgery). Any clinical alteration or complication appearing in the $24 \mathrm{~h}$ immediately after the procedure and to the time of hospital discharge was descriptively recorded.

Results are expressed as mean \pm SD. Analysis of variance was performed for repeated measures, and the $\chi^{2}$ test was performed to compare proportions. Significance was set at a $P$ value of $<0.05$. Data were processed with the SPSS statistics package (Version 8.0; SPSS Inc., Chicago, IL).

\section{Results}

Significant changes with respect to baseline values were found for heart rate, $\mathrm{CVP}, \mathrm{PaCO}_{2}$, and $\mathrm{ETCO}_{2}$. There were no significant changes for mean arterial blood pressure (MAP), $\mathrm{SaO}_{2}$, or hemoglobin (Tables 1 and 2).

Transcranial Doppler ultrasound results showed no variations at any of the four time points studied (Table 3). There were no statistically significant changes in right or left MCA blood-flow velocity or in the PI, despite significant differences in $\mathrm{ETCO}_{2}$ and $\mathrm{PaCO}_{2}$ values.

Eight of the patients included in the analysis presented moderately intense headaches during the immediate postoperative period. The characteristics of the headaches were similar in all patients-holocranial location and slightly pulsatile. All cases resolved with conservative treatment consisting of bed rest and oxygen therapy. All the patients were discharged from the hospital at the expected time, according to the criteria for surgery.

\section{Discussion}

The results of this study showed significant changes in heart rate and CVP with respect to baseline values in laparoscopic surgical patients with pneumoperitoneum and head-down positioning. Significant differences were also recorded for $\mathrm{PaCO}_{2}$ and $\mathrm{ETCO}_{2}$. Nevertheless, in contrast to the findings of other authors $(3,4)$, there were no significant changes in mean MCA blood-flow velocity.

A study by Abe et al. (3) in a series of 14 patients undergoing laparoscopic hysterectomy in head-down positioning demonstrated a significant increase in mean cerebral blood-flow velocity with respect to baseline levels. The authors did not mention whether bilateral measurements were taken to exclude inherent differences between the two vessels; however, hemodynamic variables showed no variation, in contrast to the results obtained in our study, and mean MCA blood-flow velocity was significantly increased compared with baseline. In a study by Fujii et al. (4) in a series of 10 patients undergoing laparoscopic cholecystectomy and placed in a head-up position, the methodology used was identical to that of Abe et al. 
Table 1. Results for Hemodynamic Variables

\begin{tabular}{|c|c|c|c|c|c|}
\hline Variable & Baseline & $60 \mathrm{~min}$ & $120 \mathrm{~min}$ & $180 \mathrm{~min}$ & End \\
\hline HR (bpm) & $76 \pm 14$ & $63 \pm 10^{*}$ & $63 \pm 10^{*}$ & $65 \pm 12^{*}$ & $73 \pm 16^{*}$ \\
\hline MAP (mm Hg) & $81 \pm 9$ & $90 \pm 9$ & $87 \pm 15$ & $87 \pm 12$ & $89 \pm 13$ \\
\hline CVP (mm Hg) & $13 \pm 2$ & $19 \pm 4^{*}$ & $22 \pm 5^{*}$ & $22 \pm 4^{*}$ & $20 \pm 1^{*}$ \\
\hline
\end{tabular}

$\mathrm{HR}=$ heart rate; MAP = mean arterial blood pressure; CVP = central venous pressure; baseline start of study, supine position; $60-180$ min $=$ minutes of head-down positioning and pneumoperitoneum; end = peritoneal cavity deflated and return to supine position.

${ }^{*} P<0.05$ as compared with baseline values.

Table 2. Results for Respiratory Variables

\begin{tabular}{lccccc}
\hline \multicolumn{1}{c}{ Variable } & Baseline & $60 \mathrm{~min}$ & $120 \mathrm{~min}$ & $180 \mathrm{~min}$ & End \\
\hline $\mathrm{Hb}(\mathrm{g} / \mathrm{dL})$ & $12.2 \pm 2.6$ & $12.2 \pm 2.5$ & $12.0 \pm 2.6$ & $12.0 \pm 2.3$ & $11.9 \pm 2.4$ \\
$\mathrm{PaCO}_{2}(\mathrm{~mm} \mathrm{Hg})$ & $33 \pm 2$ & $39 \pm 2^{*}$ & $42 \pm 5^{*}$ & $41 \pm 4^{*}$ & $41 \pm 3^{*}$ \\
$\mathrm{ETCO}_{2}(\mathrm{~mm} \mathrm{Hg})$ & $30 \pm 3$ & $36 \pm 5^{*}$ & $37 \pm 4^{*}$ & $37 \pm 3^{*}$ & $38 \pm 4^{*}$ \\
$\mathrm{SaO}_{2}(\mathrm{~mm} \mathrm{Hg})$ & $99 \pm 0.2$ & $99 \pm 0.3$ & $99 \pm 0.4$ & $99 \pm 0.4$ & $99 \pm 0.5$ \\
\hline
\end{tabular}

$\mathrm{Hb}=$ hemoglobin; $\mathrm{ETCO}_{2}=$ end-tidal $\mathrm{CO}$; concentration; baseline = start of study, supine position; 60-180 min = minutes of head-down positioning and pneumoperitoneum; end $=$ peritoneal cavity deflated and return to supine position.

${ }^{*} P<0.05$ as compared with baseline values.

Table 3. Results for Transcranial Doppler Ultrasound

\begin{tabular}{lccccc}
\hline Variable & Baseline & $60 \mathrm{~min}$ & $120 \mathrm{~min}$ & $180 \mathrm{~min}$ & End \\
\hline MCA (r) & $49 \pm 12$ & $41 \pm 12$ & $42 \pm 14$ & $46 \pm 8$ & $42 \pm 14$ \\
MCA (l) & $46 \pm 9$ & $46 \pm 12$ & $38 \pm 8$ & $38 \pm 6$ & $45 \pm 10$ \\
PI (r) & $0.83 \pm 0.12$ & $0.98 \pm 0.42$ & $0.84 \pm 0.13$ & $0.94 \pm 0.15$ & $0.94 \pm 0.19$ \\
PI (I) & $0.82 \pm 0.08$ & $1.06 \pm 0.39$ & $0.92 \pm 0.21$ & $0.86 \pm 0.11$ & $0.90 \pm 0.09$ \\
\hline
\end{tabular}

MCA = middle cerebral artery blood-flow velocity; $\mathrm{PI}=$ pulsatility index; $(\mathrm{r})=$ right; $(\mathrm{l})=$ left; baseline $=$ start of study, supine position; $60-180 \mathrm{~min}=$ minutes of head-down positioning and pneumoperitoneum; end = peritoneal cavity deflated and return to supine position.

(3), and the results were similar; a significant increase in mean cerebral blood-flow velocity with respect to baseline levels was observed, even though the positioning of the patients was completely different. Using methodology developed by Markwalder et al. (5) in healthy subjects, both of these studies established a regression equation between cerebral blood-flow velocity and $\mathrm{PaCO}_{2}$ and found a positive linear correlation. This contrasts with Markwalder et al.'s proposal in that the equation contemplates an exponential increase in MCA blood-flow velocity values with respect to $\mathrm{PaCO}_{2}$; thus, one might question whether the statistical method was applied properly in these works. Our results in patients undergoing lengthier pneumoperitoneum and head-down positioning did not show this linear correlation; instead, there was a significant variation in $\mathrm{PaCO}_{2}$, and there were no significant changes in MCA flow velocity-results that can be considered consistent with Markwalder et al.'s methodology.

In a series of 15 patients undergoing laparoscopic hysterectomy in a head-down position, Kirkinen et al. (6) performed a complete hemodynamic study by using flow-directed pulmonary artery catheters to assess all the variables that could alter or modify mean MCA blood-flow velocity as determined by transcranial Doppler imaging. The results from our series are comparable to those obtained by Kirkinen et al. There were no significant changes in the PI or mean blood-flow velocities in the MCA, despite significant changes in the hemodynamic variables studied.

The consequences of the surgical technique on cerebral hemodynamics are not evaluable from the clinical standpoint $(3,4,6)$, and we did not find significant variations in the Doppler variables investigated; however, we did find other hemodynamic changes, particularly in ICP. Experimental studies in animals and only two case reports in patients with neurological pathology are available on this subject $(7,8)$. One involved a 37-year-old patient diagnosed with a slowgrowing temporal lobe glioma who required laparoscopic cholecystectomy. ICP was monitored with a Camino $^{\circledR}$ intraparenchymal fiberoptic sensor. Increased ICP with respect to the baseline value was registered with both head-down and head-up positioning of the patient (7). The second report included an 18-year-old patient with a ventriculoperitoneal shunt as a sequela of posttraumatic subdural hematoma, who also required laparoscopic cholecystectomy. In this case, the authors also reported a significant increase in ICP after $\mathrm{CO}_{2}$ insufflation (8).

The various studies performed in experimental animals provide insight into the mechanisms involved. The first work on this subject was done by Josephs et al. (9) in a porcine model. The authors assessed ICP in healthy animals after pneumoperitoneum and in the same animals after artificially increasing the ICP with 
a Foley probe in the epidural space of the parietooccipital region (head injury model). The animals were placed in a $30^{\circ}$ head-up position, and IAP was $15 \mathrm{~mm}$ $\mathrm{Hg}$. After establishing the pneumoperitoneum, the authors found an increase in ICP that was independent from the remaining hemodynamic and respiratory variables (MAP, $\mathrm{pH}, \mathrm{PaCO}_{2}$, and $\mathrm{Po}_{2}$ ), suggesting that a mechanical effect was the main cause of the increase in ICP. The authors implicated two mechanisms in this change:

1. Decreased thoracoabdominal compliance due to the pneumoperitoneum provokes an increase in intrathoracic pressure. This, in turn, leads to a reduction in cerebral venous return and an increase in ICP.

2. Decreased drainage of the lumbar plexuses can increase spinal canal pressure and thereby increase cerebrospinal fluid pressure and ICP.

Rosenthal et al. $(10,11)$ presented another theory to explain the repercussions of pneumoperitoneum at the cranial level. Using the same methods as Josephs et al. (9), the authors studied ICP variations in animals placed in different head positions. Five animals were successively placed in supine decubitus, head-up, and head-down positions. IAPs were $0,8,16$, and $24 \mathrm{~mm}$ $\mathrm{Hg} . \mathrm{PaCO}_{2}$ remained stable at $35-45 \mathrm{~mm} \mathrm{Hg}$. The authors observed a significant increase in ICP after pneumoperitoneum in both the head-down and head-up positions, with an earlier increase in the former, which did not change substantially when IAP was at $8-24 \mathrm{~mm} \mathrm{Hg}$. Additionally, analysis of variations in $\mathrm{PaCO}_{2}$ at several time points after the pneumoperitoneum was established showed a linear, nonsignificant increase in these values. The authors suggested that besides the mechanisms postulated by Josephs et al. (9), there is another, later mechanism, which they termed chemical (variations in $\mathrm{PaCO}_{2}$ ), that contributes to increased ICP.

Corroborating this theory, a subsequent work by this group analyzed the effects of hyper- and hypoventilation on ICP after $\mathrm{CO}_{2}$ insufflation in an animal model (11). On the basis of their results, the authors presented the following conclusions:

1. An acute increase in IAP produces an increase in ICP.

2. Two mechanisms are implicated in this association: 1) an early passive mechanical effect produced by stasis and increased cerebrospinal fluid pressure and 2) a late active chemical effect caused by arterial vasodilation in response to hypercapnia.

3. Increased IAP causes an increase in intrathoracic pressure with superior and inferior vena cava pressure.
4. Hypoventilation and hypercapnia can increase the ICP.

5. Hyperventilation and hypercapnia do not significantly decrease the ICP when a pneumoperitoneum with increased IAP $(>10 \mathrm{~mm} \mathrm{Hg})$ is established.

6. The hemodynamic changes that appear after an increased IAP, such as increased MAP, can be considered secondary to central nervous system responses activated to maintain cerebral perfusion pressure after increases in ICP.

7. Laparoscopic procedures requiring $\mathrm{CO}_{2}$ insufflation may be contraindicated in patients with suspected or known neurotraumatic lesions or cranioencephalic pathology.

8. Use of hyperventilation in patients with combined abdominal and cranial trauma does not significantly reduce ICP unless decompression of the abdominal cavity is performed.

Related experimental and clinical studies show that the cerebral hemodynamic changes occurring with $\mathrm{CO}_{2}$ insufflation have few or no clinical consequences in young, otherwise healthy subjects (12). In the presence of cranial or spinal canal compromise, however, the consequences are greater, and many authors $(7,8,11,12)$ recommend caution when using this technique in patients with such conditions. The consequences of increased IAP on cerebral homeostasis and ICP are particularly relevant for patients affected with cranial trauma or other neurological pathologies and who might require examination by the laparoscopic technique (9-12).

Although our series was composed of patients with no associated pathologies, headaches with similar characteristics were observed in 8 of the 17 patients included. From the clinical standpoint, we were surprised to see that none of the articles consulted $(3,4,6)$ mentioned the development of clinical manifestations related to the operative technique. Because cerebral hemodynamic conditions remained stable, from among the possible causes of this postoperative complication, we suggest that it may be related to the effect of plasma absorption of $\mathrm{CO}_{2}$ breakdown products in the closed abdominal cavity after electrocautery. Several studies have quantified the increase in plasma concentrations of carboxyhemoglobin and methemoglobin (13-15). Results have shown that the levels reached are not in the range of toxicity, and, thus, no clinical signs in the immediate postoperative period would be expected. The design of this study did not include carboxyhemoglobin and methemoglobin determination, but this was one of the mechanisms suspected initially as being responsible for the headaches. For this reason, we applied oxygen therapy in the immediate postoperative period; this seemed to be 
helpful, because no other measures were needed to resolve the headache.

None of the patients studied experienced other complications. One patient presented with thrombosis of the external jugular vein at seven days after the procedure, which most likely had a multifactorial etiology (16).

We conclude that lengthy laparoscopic procedures in the head-down position performed in otherwise healthy patients do not significantly affect cerebral circulation. Nevertheless, this technique should be reconsidered in patients with associated neurologic pathology when the means for proper measurement of cerebral hemodynamics are not available. Studies in larger series are necessary to assess clinical alterations that could be related to variations in cerebral hemodynamics, particularly when surgery requires lengthy head-down positioning.

The authors thank Celine L. Cavallo for translating and editing the text.

\section{References}

1. Odeberg-Wernerman S, Sollevi A. Cardiopulmonary aspects of laparoscopic surgery. Curr Opin Anaesthesiol 1996;9:529-35.

2. Pellisé F, Puig O, Rivas A, et al. Low fusion rate after L5-S1 laparoscopic anterior lumbar interbody fusion using twin standalone carbon fiber cages. Spine 2002;27:1665-9.

3. Abe K, Hashimoto N, Taniguchi A, et al. Middle cerebral artery blood flow velocity during laparoscopic surgery in head-down position. Surg Laparosc Endosc 1998;8:1-4.
4. Fujii Y, Tanaka H, Tsuruoka S, et al. Middle cerebral arterial blood flow velocity increases during laparoscopic cholecystectomy. Anesth Analg 1994;78:80-3.

5. Markwalder TM, Grolimund P, Seiler RW, et al. Dependency of blood flow velocity in the middle cerebral artery on end-tidal carbon dioxide partial pressure: a transcranial ultrasound Doppler study. J Cereb Blood Flow Metab 1984;4:368-72.

6. Kirkinen P, Hirvonen E, Kauko M, et al. Intracranial blood flow during laparoscopic hysterectomy. Acta Obstet Gynecol Scand 1995;74:71-4.

7. Irgau I, Koyfman Y, Tikellis JI. Elective intraoperative intracranial pressure monitoring during laparoscopic cholecystectomy. Arch Surg 1995;130:1011-3.

8. Lückers O, Born JD, Denoel A. Pneumopéritoine et pression intracrânienne: une relation méconnue. Rev Med Liege 1997;52: $712-4$.

9. Josephs LG, Este-McDonald JR, Birkett DH, et al. Diagnostic laparoscopy increases intracranial pressure. J Trauma 1994;36: 815-9.

10. Rosenthal RJ, Hiatt JR, Phillips EH, et al. Intracranial pressure: effects of pneumoperitoneum in a large-animal model. Surg Endosc 1997;11:376-80.

11. Rosenthal RJ, Friedman R, Chidambaran A, et al. Effects of hyperventilation and hypoventilation on $\mathrm{PaCO}_{2}$ and intracranial pressure during acute elevations of intraabdominal pressure with $\mathrm{CO}_{2}$ pneumoperitoneum: large animal observations. J Am Coll Surg 1998;187:32-8.

12. Halverson A, Buchanan R, Jacobs L, et al. Evaluation of mechanism of increased intracranial pressure with insufflation. Surg Endosc 1998;12:266-9.

13. Beebe DS, Swica H, Carlson N, et al. High levels of carbon monoxide are produced by electro-cautery of tissue during laparoscopic cholecystectomy. Anesth Analg 1993;77:338-41.

14. Wu JS, Luttmann DR, Meininger TA, et al. Production and systemic absorption of toxic byproducts of tissue combustion during laparoscopic surgery. Surg Endosc 1997;11:1075-9.

15. Wu JS, Monk T, Luttmann DR, et al. Production and systemic absorption of toxic byproducts of tissue combustion during laparoscopic cholecystectomy. J Gastrointest Surg 1998;2: 399-405.

16. Colomina MJ, Godet C, Bagó J, et al. Isolated thrombosis of the external jugular vein. Surg Laparosc Endosc 2000;4:264-7. 